\title{
Towards Quantitative 3D Chemical Analysis in TEM Using Quadrant XEDS Detector Geometry
}

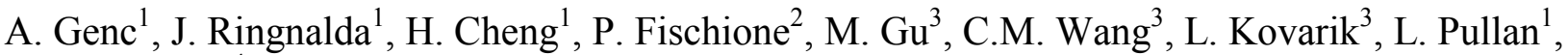 \\ and B. Freitag 4 \\ ${ }^{1}$ FEI Company, 5350 NE Dawson Creek Drive, Hillsboro, OR 97124, USA \\ ${ }^{2}$ E.A. Fischione Instruments Inc., 9003 Corporate Circle, Export, PA, 15632, USA \\ 3 Environmental Molecular Sciences Laboratory, Pacific Northwest National Laboratory, P.O. Box \\ 999, Richland, WA 99352, USA \\ ${ }^{4}$ FEI Company, Achtseweg Noord 5, P.O. Box 80066, 5600 KA, Eindhoven, The Netherlands
}

Scanning transmission electron microscopy with X-ray energy dispersive spectroscopy (STEM XEDS) allows characterization of thin foils to obtain valuable information about the microstructure and chemistry of materials at the nanometer scale. While typical thin foils used in TEM are in the 50 to 100 $\mathrm{nm}$ thickness range, important 3D structural and chemical information of the material is often overlooked. The resulting data obtained in a single orientation is convoluted throughout the analyzed thickness of the TEM foil; this lack of depth sensitivity and therefore knowledge about the structure limits the complete characterization of nanoscale materials with complex morphologies and hinders efforts to develop new materials with novel properties. To elucidate the third dimension, 3D STEM tomography techniques are of considerable interest to and have been increasingly employed by microscopists involved in the characterization of complex nanoscale materials [1,2]. Limitations to the analytical implementations of current instrumentation pose additional challenges to achieving correct and interpretable results. Despite the extensive use of TEM tomography for imaging, it is not easy to directly observe the chemistry of materials in 3D using TEM.

To overcome this problem, we employed a tomography technique for STEM XEDS which uses dynamic focus correction to allow in-focus acquisition of a STEM image with the sample tilted, the combination of symmetrically arranged four silicon drift detectors (SDD) (FEI Super-X ${ }^{\mathrm{TM}}$ ) around the sample and a Beryllium (Be) based single-tilt low background tomography holder (E.A.Fischione Instruments Inc.) that was specifically developed for the acquisition of XEDS elemental map tilt series [3]. Figurela is a schematic indicating the geometry of high-angle annular dark field (HAADF) and four XEDS detectors in STEM imaging mode. Signals from all four EDS detectors are combined into one spectrum to improve the collection efficiency. As shown in the corresponding spectra in Figure1b, compared to a conventional TEM tomography holder (graph left), the Be-based low background tomography holder (graph right) shows an improvement in spurious X-rays generated from the tomography holder as revealed by the absence of $\mathrm{Cu} \mathrm{K}$. peak in the spectra acquired at $0^{\circ}$ to $60^{\circ}$ tilt range. As indicated by the black arrows in Fig. $1 \mathrm{~b}$, the use of the Be-based low background tomography holder significantly minimizes the secondary fluorescence effects at lower X-ray energies for Ti, Cr and Co. The TEM sample is a $3 \mathrm{~mm}$ disc superalloy that consists of $\mathrm{Ni}, \mathrm{Al}, \mathrm{Ti}, \mathrm{Cr}$ and $\mathrm{Co}$ elements and does not contain any $\mathrm{Cu}$. Figure 1c shows a sub-set of the $\mathrm{Ni}\left(\mathrm{Ni}-\mathrm{K}_{\alpha}\right)$ elemental maps acquired from a $\mathrm{Li}_{1.2} \mathrm{Ni}_{0.2} \mathrm{Mn}_{0.6} \mathrm{O}_{2}$ (LNMO) nanoparticle at the tilt range of $\pm 70^{\circ}$. The use of Super- $\mathrm{X}^{\mathrm{TM}}$ detector allows tilting of the TEM sample without any shadowing effect at the positive and negative tilt directions, thus avoiding the detection problems related to specimen tilting. In this configuration, X-ray detection has a minimum dependence on the specimen tilt. Applications of 3D chemical mapping are shown in Figure 1.d, where XEDS STEM tomographic reconstructions from a LNMO nanoparticle are shown in a series of volume rendered projections [3]. 3D reconstructions illustrate 
the 3D elemental distributions of Mn (red) and $\mathrm{Ni}$ (green) in the entire nanoparticle. Compared to XEDS, HAADF STEM 3D projections indicate a relatively weaker contrast from $\mathrm{Ni}$ segregated regions in the nanoparticle due to the poor Z-contrast in LNMO.

In summary, this technique enables the successful acquisition of 3D XEDS chemical maps from $3 \mathrm{~mm}$ samples and the use of a low background tomography holder reduces the spectral artifacts typically generated in XEDS STEM tomography allowing a more quantitative analysis of the XEDS tomograms. This opens up research paths to optimize the correction for absorption and fluorescence effects that were not part of this study, but should be considered.

\section{References}

[1] P.A. Midgley etal., Ultramicroscopy 96 (2003) 413-431.

[2] P.G. Kotula et al, Microscopy and Microanalysis (2007) 13.

[3] A. Genc et al., Ultramicroscopy 108 (2008) 1603-1615.

(a)

E BEAM

(c)
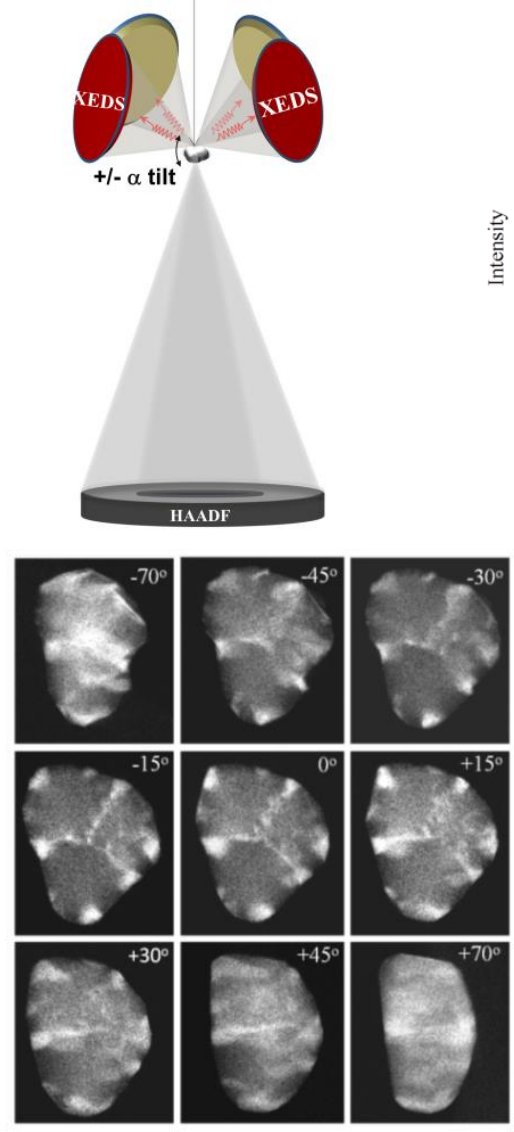

(b)

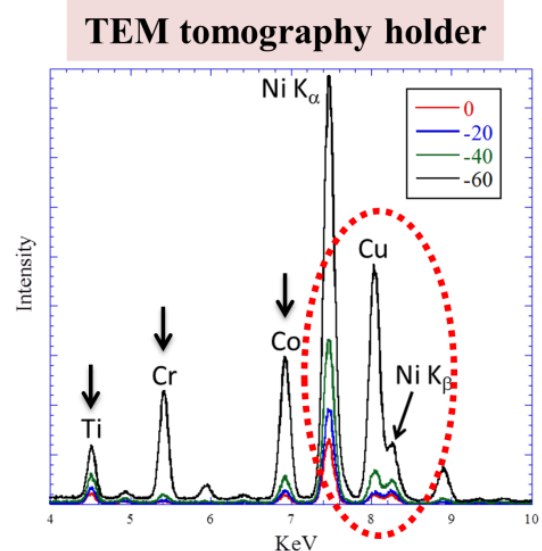

(d)

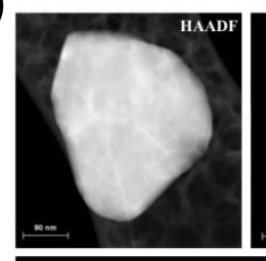

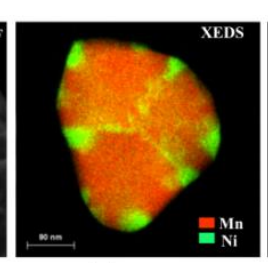

\section{Low background}

TEM tomography holder
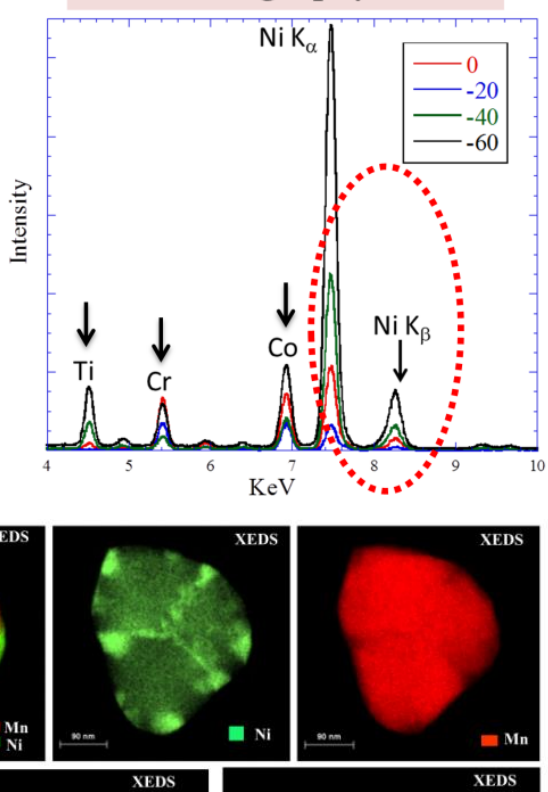
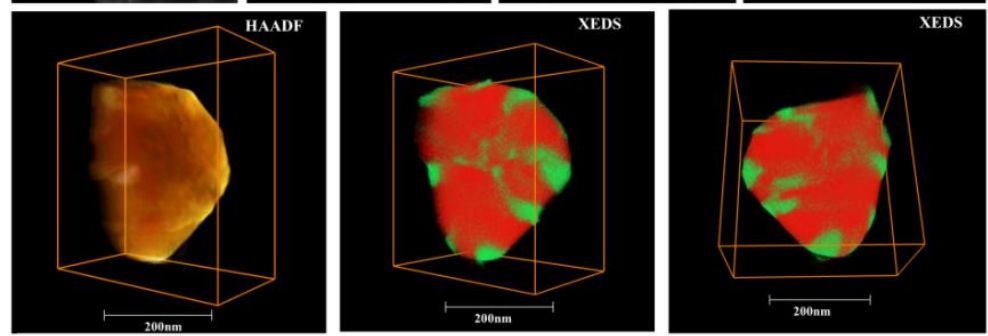

Figure 1 (a) a schematic of the symmetrically arranged four XEDS detectors around the specimen (b) comparison of tilt series spectra acquired from a conventional tomography TEM holder (graph left) and Be-based low background TEM tomography holder (graph right) (c) sub-set of the Ni $\mathrm{K}_{\alpha}$ elemental map tilt series acquired from a LNMO nanoparticle at the $\pm 70^{\circ}$ tilt range (d) comparison of HAADF and XEDS STEM of nanoparticle in 2D and 3D elemental mapping. 